\title{
The Emerging Role of Soluble Adenylyl Cyclase in Primary Biliary Cholangitis
}

\author{
Jung-Chin Chang Ulrich Beuers Ronald P.J. Oude Elferink \\ Tytgat Institute for Liver and Intestinal Research, Academic Medical Center, University of Amsterdam, \\ Amsterdam, The Netherlands
}

\section{Keywords}

Primary biliary cholangitis - Primary biliary cirrhosis .

Soluble adenylyl cyclase · Anion exchanger $2 \cdot$ Bicarbonate

Apoptosis - Barrier function

\begin{abstract}
Background: Primary biliary cholangitis (PBC; previously referred to as primary biliary cirrhosis) is a chronic fibrosing cholangiopathy with the signature of an autoimmune disease and features of intrahepatic cholestasis. Immunosuppressing treatments are largely unsuccessful. Responsiveness to ursodeoxycholic acid and reduced expression of anion exchanger 2 (AE2) on canalicular membranes and small bile ducts underline the importance of bicarbonate transportation in its disease mechanism. Soluble adenylyl cyclase (sAC; ADCY10) is an evolutionarily conserved bicarbonate sensor that regulates apoptosis, barrier function and TNF signaling. Key Messages: The biliary epithelium defends against the toxic bile by bicarbonate secretion and by maintaining a tight barrier. Passive diffusion of weak acid conjugates (e.g. bile salts and other toxins) across plasma membrane is pH-dependent. Reduced AE2 expression results in both reduced bicarbonate secretion and accumulation of bicarbonate in the cells. Increased intracellular bicarbonate leads to increased SAC activity, which regulates bile salt-induced apoptosis. Reduced bicarbonate secretion causes more bile salts to enter cells, which further increase sAC activity by releasing intracellular $\mathrm{Ca}^{2+}$ store. In vitro studies
\end{abstract}

\section{KARGER}

E-Mail karger@karger.com www.karger.com/ddi
This article is licensed under the Creative Commons AttributionNonCommercial-NoDerivatives 4.0 International License (CC BYNC-ND) (http://www.karger.com/Services/OpenAccessLicense) tribution of modified material requires written permission. demonstrate that inhibition of SAC not only corrects sensitization to bile salt-induced apoptosis as a result of AE2 downregulation but also prevents bile salt-induced apoptosis altogether. Targeting SAC is also likely to slow down disease progression by strengthening the barrier function of biliary epithelia and by reducing oxidative stress as a result of chronic inflammation. Conclusions: $\mathrm{SAC}$ is a potential therapeutic target for PBC. More in vitro and in vivo studies are needed to understand how SAC regulates bile salt-induced apoptosis and to establish its therapeutic value in PBC and other cholestatic cholangiopathies.

\section{(C) 2017 The Author(s)}

Published by S. Karger AG, Basel

The biliary tree is the passage for millimolar bile salts, xenobiotics and toxic metabolites and is arguably one of the harshest environments in the human body. The cholangiocytes, the epithelial lining of the biliary tree, is surprisingly resistant to these constant challenges. While generally less vulnerable to cholestasis than hepatocytes are, cholangiocytes are the primary target in primary biliary cholangitis (PBC). PBC is a chronic fibrosing cholangiopathy characterized by an initial presentation of fatigue and itch and cholestatic blood biochemistry, namely, increased serum alkaline phosphatase and $\gamma$-glutamyl transferase. The presence of serum anti-mitochondrial autoantibodies and the striking overrepresentation by female patients suggest that autoimmunity is at play [1]. The high concordance rate in monozygotic twins as compared to

Ronald P.J. Oude Elferink

Tytgat Institute for Liver and Intestinal Research

Academic Medical Center S1-162, Meibergdreef 69-71

NL-1105 BK Amsterdam (The Netherlands)

E-Mail r.p.oude-elferink@amc.uva.nl 
dizygotic twins $[2,3]$ and results from genome-wide association studies $[4,5]$ underline an inherited genetic predisposition. Despite features of an autoimmune disease, general immunosuppressive agents proved disappointing in PBC. Ursodeoxycholic acid (UCDA), the only US Food and Drug Administration-approved drug for PBC, improves serum liver tests and restores secretin responsiveness, but does not delay time to liver transplantation significantly and has no effect on itch and fatigue [6]. The development of an effective therapy, therefore, awaits new insights into the disease mechanism and identification of its etiology. Readers are referred to a recent review by Dyson et al. [52] for potential novel therapeutic targets in different aspects of $\mathrm{PBC}$ from aberrant immune response, cholestatic injury, fibrosis to itch and fatigue.

In this review, we summarize how recent evidence supports that soluble adenylyl cyclase (sAC; ADCY10), an evolutionarily conserved bicarbonate sensor, is an important regulator of bile salt-induced apoptosis and a potential therapeutic target in cholestatic cholangiopathies.

\section{The Bicarbonate-Responsive sAC Mediates G-Protein Independent CAMP Signaling}

sACis an evolutionarily conserved member of the mammalian adenylyl cyclase family that was relatively recently discovered. Its activity was first detected in the cytosolic fraction of adult rat testes homogenates and was found to be $\mathrm{Mn}^{2+}$-dependent and insensitive to fluoride, $\mathrm{LH}$ and FSH stimulation $[7,8]$. This was a surprising finding at that time in that the canonical mammalian adenylyl cyclase activity was known to reside solely at the plasma membrane. Only after 2 decades, the molecular identity of this enigmatic adenylyl cyclase activity in cytosol was finally cloned and characterized from rat testes, where sAC is most abundant [9]. In the human genome, the single-copied gene ADCY10 encodes an $187 \mathrm{kD}$ full-length enzyme together with many shorter isoforms as a result of extensive splicing and is expressed in a wide range of tissues [10]. The presence of sAC in human liver [11] and mouse primary cholangiocytes [12] has been demonstrated.

Although both belong to class III nucleotidyl cyclases, sAC differs from the transmembrane adenylyl cyclases (tmAC; ACDY1 ACDY9) in several aspects [13] (fig. 1). First, sAC is not regulated by $\mathrm{G}$ proteins, nor is it activated by pan-tmAC activators, such as forskolin (FSK) and fluoride [7-9]. Rather, sAC is activated by bicarbonate and fine-tuned by calcium [11, 14-16]. Second, while tmACs are localized on the plasma membrane, the sAC

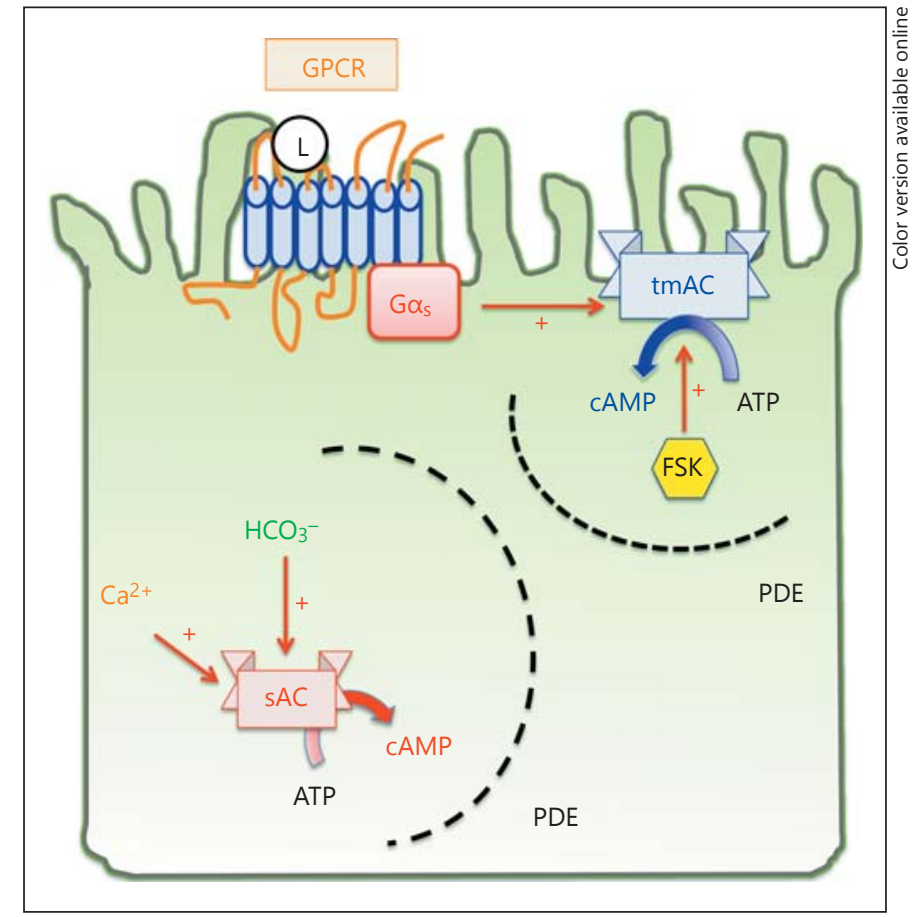

Fig. 1. Compartmentalization of intracellular cAMP signaling. The classic cAMP signaling takes place at the plasma membrane and begins with a ligand (L) binding to its GPCR on the plasma membrane. The G protein is heterotrimeric, consisting of $\alpha, \beta, \gamma$ subunit. Receptor-ligand binding triggers a conformational change and releases the a subunit of the $G$ protein. Depending on the type of $\alpha$ subunit, it can either activate $(\mathrm{Ga} s$, depicted in this figure) or inhibit $\left(\mathrm{Ga}_{\mathrm{i}}\right)$ the activity of tmAC, ADCY1 9. In contrast to tmAC signaling, sAC takes place in different subcellular compartments, including cytosol, mitochondria and nuclei. sAC is activated by bicarbonate and calcium, but not $\mathrm{G}$ protein or the pan-tmAC activator FSK. The specificity of cAMP signaling is made possible by scaffolding proteins that assemble the adenylyl cyclase, cAMP effectors (PKA and Epac), and the substrates in signaling microdomains bordered by cAMP-impermeable membranes or phosphodiesterases, which degrade cAMP.

isoforms are found in several subcellular compartments, including the cytosol [17], mitochondria [18, 19], cytoskeleton [20] and nuclei [21]. Third, as a result of its intracellular localization, sAC produces cAMP in response to intracellular fluctuations of bicarbonate and calcium, while tmACs relay the extracellular signals that activate $G$ protein-coupled receptors (GPCR).

The cAMP signaling of sAC is versatile. In the presence of carbonic anhydrase, the fluctuation in proton concentration is instantly translated into that of bicarbonate. Therefore, $\mathrm{sAC}$ is a perfect sensor for $\mathrm{pH}$ homeostasis.

In addition to its roles in $\mathrm{pH}$ homeostasis via bicarbonate sensing, sAC also regulates mitochondrial oxidative 
phosphorylation, glycogenolysis, tumor necrosis factor signaling in neutrophils, endothelial barrier function, spermatogenesis and fertilization [22, 23].

\section{The Biliary Bicarbonate Umbrella Hypothesis and Its Flip Side}

Just like the stomach and small intestines are capable of digesting food without digesting themselves, biliary epithelia are equipped with defensive mechanisms that allow the transit of the bile salts, the biological detergent, at high concentration without being dissolved. In this regard, it seems that bicarbonate is the evolutionarily favored anion for this defensive measure.

The frequent association with exocrine failure (salivary glands, lacrimal glands, pancreas, etc.) in PBC patients led Prieto, Medina et al. [24] to hypothesize that impaired bicarbonate secretion might underlie these exocrine pathologies. They found that anion exchanger 2 (AE2; SLC4A2), a chloride/bicarbonate exchanger, is downregulated in the apical membrane of both hepatocytes and cholangiocytes and in peripheral blood mononuclear cells in PBC patients [24, 25]. Consistent with the hypothesis, the expression of AE2 in the salivary glands of patients with Sjögren's syndrome is also downregulated [26]. In addition, the correction of impaired secretin-stimulated biliary bicarbonate secretion and AE2 expression on the apical membranes of hepatocytes and cholangiocytes parallels biochemical response in UCDA-responsive PBC patients [24, 27]. Furthermore, $\mathrm{AE} 2_{\mathrm{a}, \mathrm{b}}{ }^{-1-}$ mice have increased serum alkaline phosphatase and develop anti-mitochondrial antibody targeting the E2 subunit of pyruvate dehydrogenase complex as they age [28].

Recognizing the importance of bicarbonate secretion as a protective measure against bile salts, a defective 'biliary bicarbonate umbrella' was proposed as a common pathology of fibrosing cholangiopathies [29]. The protonated bile salts (BAH) are nonpolar, can enter cells by diffusion and cause apoptosis. The core concept of biliary bicarbonate umbrella hypothesis is that the biliary bicarbonate secretion serves to maintain an alkaline barrier, which renders bile salts in their polar, deprotonated and membrane-impermeant status. When the bicarbonate secretory machinery fails, as may be expected during the downregulation of AE2 expression, cholangiocytes may become vulnerable to millimolar concentrations of bile salt monomers that normally pass along the bile ducts. To test this hypothesis, Hohenester et al. [30] used the im-

Role of sAC in PBC mortalized human cholangiocytes cell line H69 (hereafter referred to as H69 cholangiocytes) as a model and demonstrated that bile salt toxicity is $\mathrm{pH}$-dependent and that knockdown of AE2 indeed sensitized $\mathrm{H} 69$ cholangiocytes to bile salt-induced apoptosis. However, AE2-knockdown $\mathrm{H} 69$ cholangiocytes were also more vulnerable to apoptosis induced by etoposide, which enters cells in a $\mathrm{pH}$-independent fashion. This unexpected finding indicates that while bicarbonate secretion might be important for keeping bile salt and other weak acid toxins out of cells, bicarbonate retention could have a more general impact on the regulation of apoptosis.

\section{sAC as a Novel Regulator of Bile Salt-Induced Apoptosis}

So how does bicarbonate retention sensitize cholangiocytes to apoptotic stimuli, if it would? Obviously the deranged homeostasis of intracellular $\mathrm{pH}$ would cause cellular distress and reduce the threshold to apoptosis, but a few observations and evidence suggest that sAC, an evolutionarily conserved bicarbonate sensor, might mediate the sensitization to apoptosis. First, fibroblasts from $A E 2_{a, b}{ }^{-1-}$ mice have an alkalinized intracellular $\mathrm{pH}$, higher cellular cAMP and higher expression of sAC at both the messenger RNA level and protein level [31]. Second, the expression of $\mathrm{sAC}$ is highest in the testes, and $A E 2_{a, b}{ }^{-/-}$ mice are infertile due to increased apoptosis in seminiferous tubules and epididymis [32]. Third, sAC is upregulated in both small and large cholangiocytes in mice during experimental cholestasis induced by either alphanaphthylisothiocyanate or lipopolysaccharide [12]. Lastly, sAC has been shown to mediate apoptosis in several models, including simulated ischemia/acidosis in coronary endothelial cells [17] and cardiomyocytes [33] and oxysterol- and oxidative stress-induced apoptosis in vascular smooth muscle cells $[34,35]$. Based on these observations from our laboratory and others, we hypothesize that sAC is involved in bile salt-induced apoptosis.

Using $\mathrm{H} 69$ cholangiocytes, we found that sAC is upregulated in AE2-knockdown human H69 cholangiocytes, recapitulating the previous finding in $A E 2_{a, b}{ }^{-l-}$ fibroblasts. Surprisingly, the inhibition of sAC with the specific inhibitor KH7, which does not inhibit tmAC, not only redeems the different susceptibility to chenodeoxycholate-induced apoptosis in wild-type and AE2-knockdown $\mathrm{H} 69$ cholangiocytes but even prevents bile salt-induced apoptosis altogether. Similarly, knocking down sAC with a lentivirus-mediated shRNA also prevents

Dig Dis 2017;35:217-223 
apoptosis induced by chenodeoxycholate. The protective effect of sAC inhibition is also present when apoptosis is induced by glycochenodeoxycholate. Importantly, chenodeoxycholate-induced apoptosis can also be prevented by sAC inhibition in primary mouse cholangiocytes [36].

Although cholangiocytes are the primary target in the initial phase of $\mathrm{PBC}$, once cholestasis develops, hepatocytes would also be affected. Bile salt-induced apoptosis in hepatocytes has been extensively characterized and has many features in common with that in cholangiocytes, including increased cytosolic free $\mathrm{Ca}^{2+}$ [37], activation of c-Jun N-terminal kinase (JNK) [38], enhancing ROS production [39], and releasing cytochrome $\mathrm{c}$ as a result of Bax-mediated permeabilization of the mitochondrial outer membrane [40]. On the basis of the similarity in signaling events induced by bile salts, it is likely that sAC also regulates bile salt-induced apoptosis in hepatocytes.

\section{Antagonism between sAC- and tmAC-Mediated cAMP Signaling}

The identification of a pro-apoptotic cAMP signaling pathway needs to be reconciled with literatures that suggest cAMP signaling to be protective in bile salt-induced apoptosis $[41,42]$. With the discovery of sAC, a cytosolic adenylyl cyclase using protein kinase A (PKA) and exchange factor directly activated by cAMP (Epac) as its cAMP effector, it becomes clear that there must be infrastructures that maintain the specificity of cAMP, a signaling molecule that readily could diffuse through the cytosol. The current theory maintains that the selectivity and specificity of cAMP are controlled by scaffolding proteins like A-kinase anchor proteins [43] and by compartmenting cAMP into microdomains bordered by the activity of abundant phosphodiesterases [44]. It follows that, cAMP produced by sAC can have a different or even opposite effect from that of the $G$ protein-regulated tmACs. In other words, the effect of cAMP is decided by the subcellular localization of the cAMP effector. This is best exemplified by the works of Sayner et al. $[45,46]$ showing that cAMP produced at plasma membrane strengthens the endothelial barrier, whereas cAMP produced within the cytosol is barrier-disruptive. Also, Kumar et al. [17] demonstrated that sAC-derived cAMP mediates acidosis-induced apoptosis in coronary endothelial cells, while stimulating tmACs by FSK is protective.

This updated concept of cAMP signaling is critical to understand why sAC-derived cAMP mediates bile saltinduced apoptosis, while tmAC-derived cAMP protects against bile salt-induced apoptosis. While cytoplasmic cAMP (derived from sAC) mediates bile salt-induced apoptosis, tmAC-derived cAMP (which can be specifically boosted by FSK stimulation) prevents bile salt-induced apoptosis. Interestingly, tmAC-derived cAMP signaling seems to dominate over sAC-derived cAMP signaling as dibutyryl-cAMP, which is membrane-permeant and activates cAMP effectors from both sAC and tmACs, inhibits bile salt-induced apoptosis. While both the activation of tmAC by FSK and inhibition of sAC by $\mathrm{KH} 7$ prevent bile salt-induced apoptosis, only FSK prevents the activation of JNK by bile salts (unpublished observation from our laboratory). This observation suggests that tmAC-derived cAMP signaling probably acts upstream of sAC-derived cAMP signaling.

This implies that the tmAC-derived cAMP possibly inhibits bile salt-induced apoptosis at a step upstream of sAC. The exact mechanism how tmAC signaling antagonizes sAC signaling remains to be investigated, but available evidence suggests that the anti-apoptotic cAMP signaling is mediated via Epac [47] while pro-apoptotic signaling by sAC is PKA-dependent $[17,35]$.

\section{Chronic Inflammation, Oxidative Stress and Barrier Function}

While bile salt-induced apoptosis is central to cholangiocyte damage in $\mathrm{PBC}$, chronic inflammation, the resulting oxidative stress and compromised barrier function all contribute to hepatobiliary damage and fibrosis. Importantly, chronic inflammation and the resulting oxidative stress are characteristic of PBC even in the early stage when cholestasis is not overt. Lipid peroxidation markers (e.g. 8-isoprostane and malondialdehyde) are increased in PBC patients and plasma glutathione level is reduced to about $30 \%$ that of normal subject $[48,49]$, which can aggravate bile salt-induced apoptosis. In addition to preventing bile salt-induced apoptosis, targeting SAC in PBC can also help to counteract damage from oxidative stress, as sAC has been shown to mediate oxidative stress-induced apoptosis in both in vivo and in vitro models [17, 33-35] and TNF-induced respiratory burst in neutrophils [50].

In addition to bicarbonate secretion, tight junction and glycocalyx on the apical membrane are both essential for forming a bile-resistant cellular lining [30]. Proliferating cholangiocytes in a chronically inflamed environment are especially vulnerable to bile salts before they repolarize and reestablish their tight junctions. Recently, Ivonnet et al. [51] demonstrated that sAC regulates hy- 
Fig. 2. An updated working model of bile salt-induced apoptosis in PBC. Bile salts are weak acid and its membrane permeability is $\mathrm{pH}$-dependent. BAH are nonpolar, membrane-permeant, and toxic. In healthy cholangiocytes, bicarbonate secretion by AE2 helps to keep bile salt in the deprotonated state (BA). In PBC, bicarbonate secretion is impaired as a result of reduced AE2 expression, which increases the entry of bile salts. Inside the cells, bile salts release $\mathrm{Ca}^{2+}$ from intracellular stores. The retained bicarbonate and increased cytosolic $\mathrm{Ca}^{2+}$ activates SAC, lowering the threshold for bile salt-induced apoptosis. Inhibition of sAC can prevent bile salt-induced apoptosis, even when AE2 is downregulated.

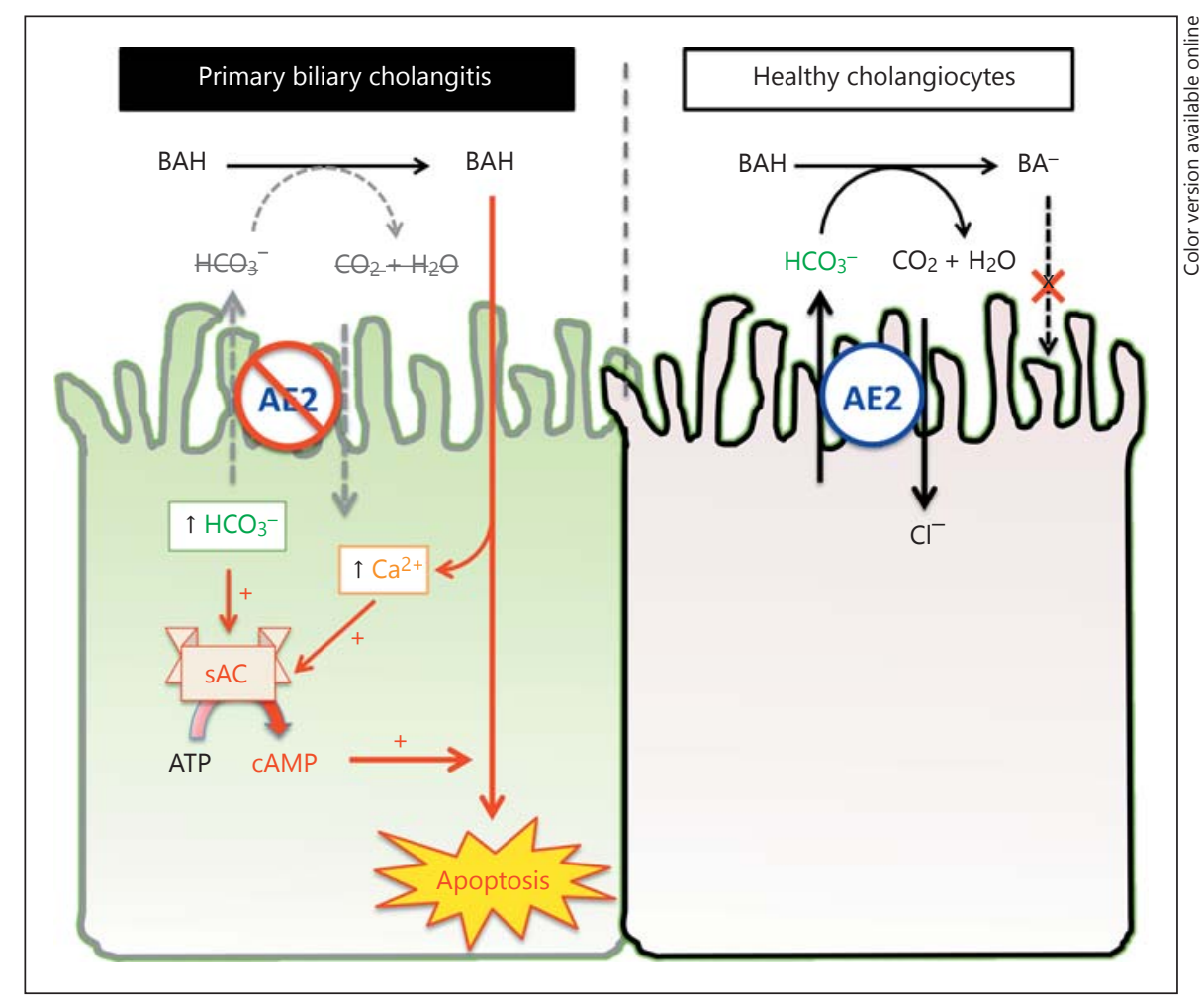

drogen peroxide-induced reduction in the barrier function, suggesting that targeting SAC can have multiple beneficial effects on PBC.

\section{Future Perspective}

Emerging evidence suggests that SAC plays a critical role in bile salt-induced apoptosis and is a potential therapeutic target in PBC and other cholestatic cholangiopathies (fig. 2). Targeting sAC has the potential to counteract apoptosis induced by bile salts, oxidative stress and other toxins and xenobiotics in bile. The compromised barrier function in chronically inflamed biliary epithelium is likely to be strengthened by inhibiting sAC. In ad- dition, sAC inhibition is likely to improve chronic inflammation, for example, by targeting TNF signaling. The role of sAC in cholestatic cholangiopathies awaits validation by in vivo experiments. Currently, most studies on SAC focus on its biochemical properties and cell biology. The physiologic functions of sAC are now in the process of being understood and more research effort is needed to explore its potential therapeutic values in diseases involving apoptosis, oxidative stress, disrupt barrier function and chronic inflammation.

\section{Disclosure Statement}

The authors have no disclosures.

\section{References}

1 Selmi C, Bowlus CL, Gershwin ME, Coppel RL: Primary biliary cirrhosis. Lancet 2011; 377:1600-1609.

2 Selmi C, Mayo MJ, Bach N, Ishibashi H, Invernizzi P, Gish RG, Gordon SC, Wright HI, Zweiban B, Podda M, Gershwin ME: Primary biliary cirrhosis in monozygotic and dizy- gotic twins: genetics, epigenetics, and environment. Gastroenterology 2004;127:485492.

-3 Chohan MR: Primary biliary cirrhosis in twin sisters. Gut 1973;14:213-214.

-4 Hirschfield GM, Liu X, Han Y, Gorlov IP, Lu Y, Xu C, Lu Y, Chen W, Juran BD, Coltescu
C, Mason AL, Milkiewicz P, Myers RP, Odin JA, Luketic VA, Speiciene D, Vincent C, Levy C, Gregersen PK, Zhang J, Heathcote EJ, Lazaridis KN, Amos CI, Siminovitch KA: Variants at IRF5-TNPO3, 17q12-21 and MMEL1 are associated with primary biliary cirrhosis. Nat Genet 2010;42:655-657. 
5 Mells GF, Floyd JA, Morley KI, Cordell HJ, Franklin CS, Shin SY, Heneghan MA, Neuberger JM, Donaldson PT, Day DB, Ducker SJ, Muriithi AW, Wheater EF, Hammond CJ, Dawwas MF; UK PBC Consortium; Wellcome Trust Case ControlConsortium 3, Jones DE, Peltonen L, Alexander GJ, Sandford RN, Anderson CA: Genome-wide association study identifies 12 new susceptibility loci for primary biliary cirrhosis. Nat Genet 2011;43: 329-332.

6 Rudic JS, Poropat G, Krstic MN, Bjelakovic G, Gluud C: Ursodeoxycholic acid for primary biliary cirrhosis. Cochrane Database Syst Rev 2012;12:CD000551.

7 Braun T, Dods RF: Development of a Mn-2+sensitive, 'soluble' adenylate cyclase in rat testis. Proc Natl Acad Sci U S A 1975;72:1097-1101.

-8 Braun T, Frank H, Dods R, Sepsenwol S: $\mathrm{Mn} 2+$-sensitive, soluble adenylate cyclase in rat testis. Differentiation from other testicular nucleotide cyclases, Biochim Biophys Acta 1977;481:227-235.

9 Buck J, Sinclair ML, Schapal L, Cann MJ, Levin LR: Cytosolic adenylyl cyclase defines a unique signaling molecule in mammals. Proc Natl Acad Sci U S A 1999;96:79-84.

10 Levin LR, Buck J: Physiological roles of acidbase sensors. Annu Rev Physiol 2015;77:347362.

11 Geng W, Wang Z, Zhang J, Reed BY, Pak CY, Moe OW: Cloning and characterization of the human soluble adenylyl cyclase. Am J Physiol Cell physiol 2005;288:C1305-C1316.

12 Strazzabosco M, Fiorotto R, Melero S, Glaser S, Francis H, Spirli C, Alpini G: Differentially expressed adenylyl cyclase isoforms mediate secretory functions in cholangiocyte subpopulation. Hepatology 2009;50:244-252.

13 Steegborn C: Structure, mechanism, and regulation of soluble adenylyl cyclases - similarities and differences to transmembrane adenylyl cyclases. Biochim Biophys Acta 2014; 1842(12 pt B):2535-2547.

14 Jaiswal BS, Conti M: Calcium regulation of the soluble adenylyl cyclase expressed in mammalian spermatozoa. Proc Natl Acad Sci U S A 2003;100:10676-10681.

-15 Litvin TN, Kamenetsky M, Zarifyan A, Buck J, Levin LR: Kinetic properties of 'soluble' adenylyl cyclase. Synergism between calcium and bicarbonate. J Biol Chem 2003;278: 15922-15926.

16 Chen Y, Cann MJ, Litvin TN, Iourgenko V, Sinclair ML, Levin LR, Buck J: Soluble adenylyl cyclase as an evolutionarily conserved bicarbonate sensor. Science 2000;289:625-628.

17 Kumar S, Kostin S, Flacke JP, Reusch HP, Ladilov Y: Soluble adenylyl cyclase controls mitochondria-dependent apoptosis in coronary endothelial cells. J Biol Chem 2009;284: 14760-14768.

18 Acin-Perez R, Salazar E, Kamenetsky M, Buck J, Levin LR, Manfredi G: Cyclic AMP produced inside mitochondria regulates oxidative phosphorylation. Cell Metab 2009;9:265276.
Di Benedetto G, Scalzotto E, Mongillo M, Pozzan T: Mitochondrial $\mathrm{Ca}^{2+}$ uptake induces cyclic AMP generation in the matrix and modulates organelle ATP levels. Cell Metab 2013;17:965-975.

20 Zippin JH, Chen Y, Nahirney P, Kamenetsky M, Wuttke MS, Fischman DA, Levin LR, Buck J: Compartmentalization of bicarbonate-sensitive adenylyl cyclase in distinct signaling microdomains. FASEB J 2003;17:82-84.

-21 Zippin JH, Farrell J, Huron D, Kamenetsky M, Hess KC, Fischman DA, Levin LR, Buck J: Bicarbonate-responsive 'soluble' adenylyl cyclase defines a nuclear cAMP microdomain. J Cell Biol 2004;164:527-534.

22 Chang JC, Oude-Elferink RP: Role of the bicarbonate-responsive soluble adenylyl cyclase in $\mathrm{pH}$ sensing and metabolic regulation. Front Physiol 2014;5:42.

23 Tresguerres M, Levin LR, Buck J: Intracellular cAMP signaling by soluble adenylyl cyclase. Kidney Int 2011;79:1277-1288.

24 Medina JF, Martínez-Ansó, Vazquez JJ, Prieto J: Decreased anion exchanger 2 immunoreactivity in the liver of patients with primary biliary cirrhosis. Hepatology 1997;25:12-17.

25 Prieto J, Qian C, García N, Díez J, Medina JF: Abnormal expression of anion exchanger genes in primary biliary cirrhosis. Gastroenterology 1993;105:572-578.

26 Vázquez JJ, Vázquez M, Idoate MA, Montuenga L, Martínez-Ansó E, Castillo JE, García N, Medina JF, Prieto J: Anion exchanger immunoreactivity in human salivary glands in health and Sjögren's syndrome. Am J Pathol 1995; 146:1422-1432.

27 Prieto J, Garcia N, Martí-Climent JM, Peñuelas I, Richter JA, Medina JF: Assessment of biliary bicarbonate secretion in humans by positron emission tomography. Gastroenterology 1999;117:167-172.

28 Salas JT, Banales JM, Sarvide S, Recalde S, Ferrer A, Uriarte I, Oude Elferink RP, Prieto J, Medina JF: Ae2a,b-deficient mice develop antimitochondrial antibodies and other features resembling primary biliary cirrhosis. Gastroenterology 2008;134:1482-1493.

29 Beuers U, Hohenester S, de Buy Wenniger LJ, Kremer AE, Jansen PL, Elferink RP: The biliary $\mathrm{HCO}(3)(-)$ umbrella: a unifying hypothesis on pathogenetic and therapeutic aspects of fibrosing cholangiopathies. Hepatology 2010;52:1489-1496.

30 Hohenester S, Wenniger LM, Paulusma CC, van Vliet SJ, Jefferson DM, Elferink RP, Beuers U: A biliary HCO3- umbrella constitutes a protective mechanism against bile acid-induced injury in human cholangiocytes. Hepatology 2012;55:173-183.

-31 Mardones P, Medina JF, Elferink RP: Activation of cyclic AMP Signaling in Ae2-deficient mouse fibroblasts. J Biol Chem 2008;283: 12146-12153.

32 Medina JF, Recalde S, Prieto J, Lecanda J, Saez E, Funk CD, Vecino P, van Roon MA, Ottenhoff R, Bosma PJ, Bakker CT, Elferink RP: Anion exchanger 2 is essential for spermio- genesis in mice. Proc Natl Acad Sci U S A 2003;100:15847-15852.

-33 Appukuttan A, Kasseckert SA, Micoogullari M, Flacke JP, Kumar S, Woste A, Abdallah Y, Pott L, Reusch HP, Ladilov Y: Type 10 adenylyl cyclase mediates mitochondrial Bax translocation and apoptosis of adult rat cardiomyocytes under simulated ischaemia/reperfusion. Cardiovasc Res 2012;93:340-349.

- 34 Appukuttan A, Kasseckert SA, Kumar S, Reusch HP, Ladilov Y: Oxysterol-induced apoptosis of smooth muscle cells is under the control of a soluble adenylyl cyclase. Cardiovasc Res 2013;99:734-742.

35 Kumar S, Appukuttan A, Maghnouj A, Hahn S, Peter Reusch H, Ladilov Y: Suppression of soluble adenylyl cyclase protects smooth muscle cells against oxidative stress-induced apoptosis. Apoptosis 2014;19:1069-1079.

36 Chang JC, Go S, de Waart DR, Munoz-Garrido P, Beuers U, Paulusma CC, Oude Elferink $\mathrm{R}$ : Soluble adenylyl cyclase regulates bile saltinduced apoptosis in human cholangiocytes. Hepatology 2016;64:522-534.

37 Spivey JR, Bronk SF, Gores GJ: Glycochenodeoxycholate-induced lethal hepatocellular injury in rat hepatocytes. Role of ATP depletion and cytosolic free calcium. J Clin Invest 1993;92:17-24.

- 38 Reinehr R, Becker S, Keitel V, Eberle A, Grether-Beck S, Häussinger D: Bile salt-induced apoptosis involves NADPH oxidase isoform activation. Gastroenterology 2005; 129:2009-2031.

39 Rodrigues CM, Fan G, Ma X, Kren BT, Steer CJ: A novel role for ursodeoxycholic acid in inhibiting apoptosis by modulating mitochondrial membrane perturbation. J Clin Invest 1998;101:2790-2799.

-40 Rodrigues CM, Ma X, Linehan-Stieers C, Fan G, Kren BT, Steer CJ: Ursodeoxycholic acid prevents cytochrome $\mathrm{c}$ release in apoptosis by inhibiting mitochondrial membrane depolarization and channel formation. Cell Death Differ 1999;6:842-854.

41 Webster CR, Usechak P, Anwer MS: cAMP inhibits bile acid-induced apoptosis by blocking caspase activation and cytochrome $\mathrm{c}$ release. Am J Physiol Gastrointest Liver Physiol 2002;283:G727-G738.

42 Webster CR, Anwer MS: Cyclic adenosine monophosphate-mediated protection against bile acid-induced apoptosis in cultured rat hepatocytes. Hepatology 1998;27:1324-1331.

43 Wong W, Scott JD: AKAP signalling complexes: focal points in space and time. Nat Rev Mol Cell Biol 2004;5:959-970.

44 Kamenetsky M, Middelhaufe S, Bank EM, Levin LR, Buck J, Steegborn C: Molecular details of cAMP generation in mammalian cells: a tale of two systems. J Mol Biol 2006;362: 623-639.

45 Sayner SL, Alexeyev M, Dessauer CW, Stevens T: Soluble adenylyl cyclase reveals the significance of cAMP compartmentation on pulmonary microvascular endothelial cell barrier. Circ Res 2006;98:675-681. 
-46 Sayner SL, Frank DW, King J, Chen H, VandeWaa J, Stevens T: Paradoxical cAMPinduced lung endothelial hyperpermeability revealed by Pseudomonas aeruginosa ExoY. Circ Res 2004;95:196-203.

47 Johnston A, Ponzetti K, Anwer MS, Webster CR: cAMP-guanine exchange factor protection from bile acid-induced hepatocyte apoptosis involves glycogen synthase kinase regulation of c-Jun NH2-terminal kinase. Am J Physiol Gastrointest Liver Physiol 2011;301:G385-G400.

-48 Sorrentino P, Terracciano L, D’Angelo S, Ferbo U, Bracigliano A, Tarantino L, Perrella A,
Perrella O, De Chiara G, Panico L, De Stefano N, Lepore M, Mariolina, Vecchione R: Oxidative stress and steatosis are cofactors of liver injury in primary biliary cirrhosis. J Gastroenterol 2010;45:1053-1062.

49 Aboutwerat A, Pemberton PW, Smith A, Burrows PC, McMahon RF, Jain SK, Warnes TW: Oxidant stress is a significant feature of primary biliary cirrhosis. Biochim Biophys Acta 2003; 1637:142-150.

50 Han H, Stessin A, Roberts J, Hess K, Gautam N, Kamenetsky M, Lou O, Hyde E, Nathan N, Muller WA, Buck J, Levin LR, Nathan
C: Calcium-sensing soluble adenylyl cyclase mediates TNF signal transduction in human neutrophils. J Exp Med 2005;202: 353-361.

51 Ivonnet P, Unwalla H, Salathe M, Conner GE: Soluble adenylyl cyclase mediates hydrogen peroxide-induced changes in epithelial barrier function. Respir Res 2016;17:15.

52 Dyson JK, Hirschfield GM, Adams DH, Beuers U, Mann DA, Lindor KD, Jones DE: Novel therapeutic targets in primary biliary cirrhosis. Nat Rev Gastroenterol Hepatol 2015;12:147-158. 